\title{
A!
}

This is an electronic reprint of the original article.

This reprint may differ from the original in pagination and typographic detail.

Puska, M. J.; Nieminen, R. M.; Manninen, M.

\section{Electronic polarizability of small metal spheres}

Published in:

Physical Review B

DOI:

10.1103/PhysRevB.31.3486

Published: 15/03/1985

Document Version

Publisher's PDF, also known as Version of record

Please cite the original version:

Puska, M. J., Nieminen, R. M., \& Manninen, M. (1985). Electronic polarizability of small metal spheres. Physical Review B, 31(6), 3486-3495. https://doi.org/10.1103/PhysRevB.31.3486

This material is protected by copyright and other intellectual property rights, and duplication or sale of all or part of any of the repository collections is not permitted, except that material may be duplicated by you for your research use or educational purposes in electronic or print form. You must obtain permission for any other use. Electronic or print copies may not be offered, whether for sale or otherwise to anyone who is not an authorised user. 


\title{
Electronic polarizability of small metal spheres
}

\author{
M. J. Puska \\ Laboratory of Physics, Helsinki University of Technology, SF-02150 Espoo, Finland \\ R. M. Nieminen \\ Department of Physics, University of Jyväskylä, SF-40100 Jyväskylä, Finland \\ M. Manninen \\ Laboratory of Physics, Helsinki University of Technology, SF-02150 Espoo, Finland
}

(Received 28 September 1984)

\begin{abstract}
We present the results of calculations for the ground-state electron structure, static polarizability, and dynamic response of small metal (jellium) spheres in vacuum or embedded in a dielectric. Fully self-consistent time-dependent density-functional methods are used. In particular, the static and dynamic responses to an incident electric field (dipolar polarizability and photoabsorption) are obtained. The results show substantial deviations from either classical or approximate quantummechanical solutions, and provide reference data for simplified treatments.
\end{abstract}

\section{INTRODUCTION}

The electronic and optical properties of small metal particles and their aggregates have attracted much interest during the last decade. ${ }^{1-19}$ One facet of this interest stems from the large surface-to-volume ratio of small particles. On the one hand, this enhances surface-associated effects in various experimental investigations; on the other, small clusters are extensively used for mimicking surfaces and adsorbates in theoretical electronic structure calculations. However, the properties of small particles can differ drastically from those of semi-infinite substrates, when the particle linear dimension is comparable to other characteristic lengths, such as the electron de Broglie wavelength or the wavelength of incident electromagnetic radiation. They are then expected to show considerable deviations from classical behavior and size effects, which are reflected in a number of observations.

Although the problem of small metal spheres and their response to, e.g., an external electric field have received considerable attention, very few exact results exist. In this paper we report on results of an essentially exact study of a simple but qualitatively valuable model system, the jellium sphere. We consider a sphere of homogeneous background density $n_{0}$, containing a fixed number $N$ of electrons, embedded in a dielectric medium with the dielectric constant $\epsilon$. We solve the ground-state electronic structure using self-consistent density-functional methods ${ }^{20,21}$ and evaluate the static and dynamic random-phaseapproximation (RPA) response functions of the system using the spectral techniques introduced by Stott and Zaremba. $^{22}$ In particular, we calculate the static and dynamic dipole polarizability and absorbtivity of jellium spheres. Thus the calculations contain both of the two essential features of the small-particle problem: the quantum-mechanical level discreteness and the nonlocal electron response. These are tied together by the densityfunctional formalism, which incorporates the electronic exchange and correlation in terms of an effective selfconsistent potential. By extending the density-functional formalism to the time-dependent regime, one can obtain the dynamic response as well. Thus the results serve a similar purpose for the spherical particles as those of Feibelman ${ }^{23}$ for the planar jellium surface. We compare our results with those of more approximate approaches, such as classical electrodynamics, semiclassical and quantum infinite barrier model and the Thomas-Fermi model. While this paper was being written, we became aware of similar work by Ekardt, ${ }^{4,5}$ whose results agree with ours where comparison is possible. The implications of the model results for experimental observations are also briefly discussed.

The organization of the paper is as follows. In Sec. II we present the basic theory for the electronic structure, response functions and polarizability. The numerical results are given in Sec. III, where the main emphasis is placed on (i) size effects in the static polarizability, (ii) effect of the embedding dielectric, and (iii) the photoabsorption spectrum. Section IV contains a short summary.

\section{THEORY}

\section{A. Ground-state electronic structure}

The small metallic particles are mimicked in the model calculations of this work by jellium spheres with a rigid positive charge distribution,

$$
n_{+}(\mathbf{r})=n_{0} \Theta(R-r),
$$

where $R$ defines the sphere radius and $n_{0}$ is the mean valence electron density of the corresponding bulk metal. Introducing the usual density parameter $r_{s}=\sqrt[3]{3 /\left(4 \pi n_{0}\right)}$, and requiring that the sphere is neutralized by $N$ valence electrons, the sphere radius is determined by

$$
R=\sqrt[3]{N} r_{s}
$$


The ground-state electron structure is obtained by solving the Kohn-Sham equations (atomic Bohr units are used throughout), ${ }^{20,21}$

$$
\begin{aligned}
& {\left[-\frac{\nabla^{2}}{2}+v_{\mathrm{eff}}[\mathbf{r}, n(\mathbf{r})]\right] \psi_{i}(\mathbf{r})=\epsilon_{i} \psi_{i},} \\
& n(\mathbf{r})=\sum_{i=1}^{N}\left|\psi_{i}(\mathbf{r})\right|^{2}, \\
& v_{\mathrm{eff}}(\mathbf{r})=\phi(\mathbf{r})+v_{\mathrm{xc}}[n(\mathbf{r})] .
\end{aligned}
$$

$\psi_{i}(r)$ and $\epsilon_{i}$ are the Kohn-Sham single-particle eigenfunctions and eigenenergies, respectively. They are used to construct the total electron density $n$ (r) [Eq. (4)] and the minimal total energy $E_{\text {tot }}(N)$ of the cluster. The effective potential $v_{\text {eff }}$ in Eq. (3) consists of the electrostatic Hartree potential $\phi(\mathbf{r})$ and of the exchange-correlation potential $v_{\mathrm{xc}}[n(\mathbf{r})]$ for which we have invoked the standard local-density approximation using the GunnarssonLundqvist $^{24}$ energy functional $E_{\mathrm{xc}}$. Equations (3)-(5) constitute a spin-independent formalism. The generalization for a spin-dependent form is straightforward but in the present work we focus on the spin-independent case.

The electrostatic potential $\phi(r)$ in Eq. (5) satisfies the Poisson equation. If the metal sphere is surrounded by a homogeneous, insulating medium with the dielectric constant $\epsilon$, this reads

$$
\begin{aligned}
\nabla^{2} \phi & =-4 \pi\left[n(\mathbf{r})-n_{0}\right], \quad r \leq R \\
& =-\frac{4 \pi}{\epsilon} n(\mathbf{r}), \quad r>R
\end{aligned}
$$

and the proper boundary condition at the interface is

$$
\left[\frac{\partial \phi}{\partial r}\right]_{r=R}^{\text {in }}=\epsilon\left(\frac{\partial \phi}{\partial r}\right]_{r=R}^{\text {out }}
$$

where in and out refer to inside and outside derivatives at
$r=R$, respectively. Due to the spherical symmetry of the cluster, Eqs. (3)-(6) can be solved self-consistently using numerical techniques similar to those developed for free atoms.

There are useful sum rules, basically variants of the Hellmann-Feynman theorem, which should be obeyed by the self-consistent energy-minimizing solution and can thus be used to check the numerical quality of the calculations. These have been discussed for various jellium geometries by Ziesche and Lehmann. ${ }^{25}$ Another closely related theorem is the electrostatic force sum rule of Sorbello, ${ }^{7}$ stating that a uniform translation of the sphere in a homogeneous electric field requires no energy. This can be transformed into a sum rule for the static response function discussed in the next chapter. We find that our self-consistently converged results obey these sum rules to a high accuracy.

\section{B. Response function and polarizability}

The linear-response theory asserts that the Fourier component $\delta v_{\text {ext }}(\mathbf{r}, \omega)$ of an external time-dependent potential perturbation produces a density fluctuation $\delta n(r, \omega)$, which are related through

$$
\delta n(\mathbf{r}, \omega)=\int d \mathbf{r}^{\prime} \chi\left(\mathbf{r}, \mathbf{r}^{\prime}, \omega\right) \delta v_{\mathrm{ext}}\left(\mathbf{r}^{\prime}, \omega\right) .
$$

Here $\chi\left(\mathbf{r}, \mathbf{r}^{\prime}, \omega\right)$ is the frequency-dependent response function for the interacting electron system. As has been discussed by several authors, ${ }^{20,26}$ the Kohn-Sham system responds as a set of independent particles to (timedependent) changes in the effective potential $V_{\text {eff }}(r)$. Thus one can write as well

$$
\delta n(\mathbf{r}, \omega)=\int d \mathbf{r}^{\prime} \chi^{0}\left(\mathbf{r}, \mathbf{r}^{\prime}, \omega\right) \delta v_{\text {eff }}\left(\mathbf{r}^{\prime}, \omega\right),
$$

where $\chi_{0}\left(\mathbf{r}, \mathbf{r}^{\prime}, \omega\right)$ is the response function (susceptibility) for the noninteracting system, represented by the set of Kohn-Sham orbitals. The changes in the effective and external potentials are related through

$$
\delta v_{\mathrm{eff}}(\mathbf{r}, \omega)=\delta v_{\mathrm{ext}}(\mathbf{r}, \omega)+\int d \mathbf{r}^{\prime}\left(\frac{1}{\left|\mathbf{r}-\mathbf{r}^{\prime}\right|}+\frac{\delta^{2} E_{\mathrm{xc}}}{\delta n(\mathbf{r}) \delta n\left(\mathbf{r}^{\prime}\right)}\right) \delta n\left(\mathbf{r}^{\prime}, \omega\right) .
$$

Inserting this into Eq. (9), comparison with Eq. (8) leads to the integral equation for $\chi\left(\mathbf{r}, \mathbf{r}^{\prime}, \omega\right)$ :

$$
\chi\left(\mathbf{r}, \mathbf{r}^{\prime}, \omega\right)=\chi^{0}\left(\mathbf{r}, \mathbf{r}^{\prime}, \omega\right)+\iint d \mathbf{r}_{1} d \mathbf{r}_{2} \chi^{0}\left(\mathbf{r}, \mathbf{r}_{1}, \omega\right)\left(\frac{1}{\left|\mathbf{r}_{1}-\mathbf{r}_{2}\right|}+\frac{\delta^{2} E_{\mathrm{xc}}}{\delta n\left(\mathbf{r}_{1}\right) \delta n\left(\mathbf{r}_{2}\right)}\right) \chi\left(\mathbf{r}_{2}, \mathbf{r}^{\prime}, \omega\right) .
$$

The local-density approximation for exchange and correlation immediately implies

$$
\frac{\delta^{2} E_{\mathrm{xc}}}{\delta n\left(\mathbf{r}_{1}\right) \delta n\left(\mathbf{r}_{2}\right)}=\left.\delta\left(\mathbf{r}_{1}-\mathbf{r}_{2}\right) \frac{d v_{\mathrm{xc}}(n)}{d n}\right|_{n=n\left(\mathbf{r}_{1}\right)} .
$$

Note that Eq. (12) also tacitly assumes that the same (ground-state) exchange-correlation energy functional is valid for all frequencies, i.e., for the excited states as well. This is not necessarily true, but the good results obtained using this approximation for the photoabsorption spec- trum $^{26}$ suggest that the error introduced is probably quite small and certainly acceptable in a model study such as the present one. The local-density approximation for exchange and correlation also excludes "image charge" contributions, i.e., decays exponentially rather than with a Coulomb tail outside the sphere. Nonlocal and energydependent corrections may become necessary for accurate calculations for realistic molecular systems, but do not affect the qualitative features which are the main concern here.

The key system-dependent input, i.e., the independent- 
particle response function $\chi_{0}^{0}\left(\mathbf{r}, \mathbf{r}^{\prime}, \omega\right)$ can be conveniently expressed $^{26}$ in terms of the Green's functions $G\left(\mathbf{r}, \mathbf{r}^{\prime}, E\right)$ as

$$
\begin{aligned}
\chi_{0}\left(\mathbf{r}, \mathbf{r}^{\prime}, \omega\right)= & \sum_{\substack{i \\
\text { occ }}} \psi_{i}^{*}(\mathbf{r}) \psi_{i}\left(\mathbf{r}^{\prime}\right) G\left(\mathbf{r}, \mathbf{r}^{\prime}, \epsilon_{i}+\omega\right) \\
& +\sum_{\substack{i \\
\text { occ }}} \psi_{i}(\mathbf{r}) \psi_{i}^{*}\left(\mathbf{r}^{\prime}\right) G^{*}\left(\mathbf{r}, \mathbf{r}^{\prime}, \epsilon_{i}-\omega\right),
\end{aligned}
$$

where the summations run over the occupied Kohn-Sham eigenstates $i$, and the Green's function satisfies the equation

$$
\left[-\frac{1}{2} \nabla^{2}+v_{\text {eff }}(\mathbf{r})-E\right] G\left(\mathbf{r}, \mathbf{r}^{\prime}, E\right)=-\delta\left(\mathbf{r}-\mathbf{r}^{\prime}\right),
$$

with the appropriate (outgoing-wave) boundary conditions. For a rotationally invariant problem such as ours, it is particularly useful to express $\chi_{0}\left(\mathbf{r}, \mathbf{r}^{\prime}, \omega\right)$ and $G\left(\mathbf{r}, \mathbf{r}^{\prime}, E\right)$ as spherical-harmonics expansions,

$$
\chi^{0}\left(\mathbf{r}, \mathbf{r}^{\prime}, \omega\right)=\sum_{l m} \chi_{l}\left(r, r^{\prime}, \omega\right) Y_{l m}(\hat{r}) Y_{l m}\left(\hat{r}^{\prime}\right),
$$

and

$$
G\left(\mathbf{r}, \mathbf{r}^{\prime}, \omega\right)=\sum_{l m} G_{l}\left(r, r^{\prime}, E\right) Y_{l m}(\hat{r}) Y_{l m}\left(\hat{r}^{\prime}\right) .
$$

Consider now the case of an external electromagnetic field incident on the sphere. In the relevant frequency range (ultraviolet and below) the wavelength is large compared to the radius. In the nonretarded limit only the electric field couples to the electrons, and the perturbation can be written as

$$
\delta v_{\mathrm{ext}}(\mathbf{r}, t)=\mathbf{E}_{0} \cdot \mathrm{r} e^{-i \omega t},
$$

where $\mathbf{E}_{0}$ is the electric field amplitude. Then the only component that couples to the perturbation is the dipolar $(l=l)$. The noninteracting dipolar susceptibility reads

$$
\begin{aligned}
\chi^{0}\left(r, r^{\prime}, \omega\right)=\frac{1}{2 \pi} \sum_{\substack{i \\
\text { occ }}} R_{n_{i} l_{i}}(r) R_{n_{i} l_{i}}\left(r^{\prime}\right)\left[\left(l_{i}+1\right) G_{l_{i}+1}\left(r, r^{\prime}, \epsilon_{i}+\omega\right)\right. \\
\\
\left.+l_{i} G_{l_{i}-1}\left(r, r^{\prime}, \epsilon_{i}+\omega\right)+\left(l_{i}+1\right) G_{l_{i}+1}^{*}\left(r, r^{\prime}, \epsilon_{i}-\omega\right)+l_{i} G_{l-1}^{*}\left(r, r^{\prime}, \epsilon_{i}-\omega\right)\right],
\end{aligned}
$$

where $R_{n_{1} l_{i}}(r)$ is the radial part of the Kohn-Sham single-particle eigenstate $\psi_{i}$. Equation (17) assumes closed shells in spherical symmetry and spin-compensation. However, we have applied it also for open-shell situations, using the spin-independent formalism and assuming a spherically symmetric charge density for the open shell. In other words, (energy-minimizing) fractional occupation numbers for the states of the open shell are used. The Green's function satisfying Eq. (14) with principal quantum number $n$ and angular momentum $l$ has the representation

$$
G_{l}\left(r, r^{\prime}, E_{n l}\right)=\frac{j_{l}\left(r_{<}, E_{n l}\right) h_{l}\left(r_{>}, E_{n l}\right)}{W\left[j_{l}, h_{l}\right]},
$$

where $j_{l}$ and $h_{l}$ are the radial parts of the solutions to Eq. (14), and $r_{<}\left(r_{>}\right)$is the lesser (greater) of $r$ and $r^{\prime} . j_{l}$ is regular at origin and $h_{l}$ satisfies the outgoing-wave boundary condition. The Wronskian $W\left[j_{l}, h_{l}\right]$ is defined as

$$
W\left[j_{l}, h_{l}\right]=r^{2}\left(j_{l} h_{l}^{\prime}-j_{l}^{\prime} h_{l}\right),
$$

and is independent of $r$ (prime denotes differentiation with respect to $r$ ).

The dipolar response function $\chi_{l}\left(r, r^{\prime}, \omega\right)$ can now be solved from an integral equation, to which Eq. (11) is reduced and which involves only double integration over the magnitudes $r_{1}$ and $r_{2}$. Discretizing these in a finite mesh converts Eq. (11) into a matrix equation which can be handled by conventional techniques.

The induced density $\delta n(r, \omega)$ can now be calculated from Eq. (8). It is convenient to define the induced dipole-moment density $\alpha(r, \omega)$ via

$$
\delta n(\mathbf{r}, \omega)=-\alpha(r, \omega) \mathbf{r} \cdot \mathbf{E}_{0},
$$

and the total polarizability $\alpha(\omega)$ using the total induced dipole moment $\mathbf{p}$,

$$
\mathbf{p}(\omega)=-\int d \mathbf{r} \mathbf{r} \delta n(\mathbf{r}, \omega)=\alpha(\omega) \mathbf{E}_{0},
$$

or

$$
\alpha(\omega)=\frac{4}{3} \pi \int_{0}^{\infty} d r r^{3} \alpha(r, \omega)
$$

In general, $\alpha(r, \omega)$ and $\alpha(\omega)$ are complex quantities. The imaginary part of the $\alpha(\omega)$ is related to the photoabsorption cross section $\sigma(\omega)$ by

$$
\sigma(\omega)=4 \pi \frac{\omega}{c} \operatorname{Im} \alpha(\omega)
$$

The imaginary part of $\alpha(\omega)$ fulfills the sum rule

$$
\frac{2}{\pi} \int_{0}^{\infty} d \omega \frac{1}{\omega} \operatorname{Im}[\alpha(\omega)]=\alpha(\omega=0),
$$

which is a special case of the general Kramers-Kronig relations.

\section{RESULTS}

\section{A. Ground-state electron structure}

We have chosen as a representative system small jellium spheres with the density parameter $r_{s}=3.25 a_{0}\left(a_{0}=\mathrm{Bohr}\right.$ radius). This value corresponds to the nominal valence electron density in $\mathrm{Li}$ metal, which represents an intermediate value among the metallic valence electron densities. We have also performed some calculations using $r_{s}=2.07 a_{0}$ corresponding to the high-electron-density 
metal Al. Figure 1 shows for the $\mathrm{Li}$ spheres in vacuum $(\epsilon=1)$ the occupied electron energy level scheme as a function of the number of electrons in the sphere. The dashed lines between $N=68$ and 92 indicate that we have not performed calculations for this region. This is because there is no well-defined ground state corresponding either to a closed $3 s$ or $1 h$ shell. If the $3 s$ level is filled, the $1 h$ energy eigenvalue is lowered below the $3 s$ value, and vice versa. Proper energy minimization would then lead to fractional occupation numbers. This peculiarity of the local-density approximation for exchange and correlation is similar to the case encountered ${ }^{27}$ for the transition metal atoms $\mathrm{Fe}$ and $\mathrm{Co}$.

In Fig. 1 the highest occupied level in each case is shown darker and the magnitude of its eigenvalue gives a rough estimate (Koopmans theorem) for the work function (ionization potential). A more accurate determination requires the self-consistent calculation of the total energies of the neutral and singly ionized jellium spheres.

The ground-state electronic structure, ionization potentials and affinities of small metallic spheres have recently been calculated by similar techniques., ${ }^{4,8}$ We obtain generally good agreement with the results of those studies, and limit ourselves to making only a few additional comments here. Figure 1 also gives overly high orbital eigenvalues and consequently overly low work-function estimates due to the electron self-interaction inherent in the local-density approximation for exchange and correlation. However, the prominent feature (shown by the dark curves) of the work function are the large oscillations due to the shell structure. A small work function corresponds to an odd electron above closed shells, with the work function increasing along with the filling of the uppermost level. The jump at the opening of a new shell is around $1.5 \mathrm{eV}$ for the smallest spheres, decreases as the size of the sphere increases, and the work function stabilizes around the value for semi-infinite jellium given in the figure. Note that the highest occupied level in the ex-

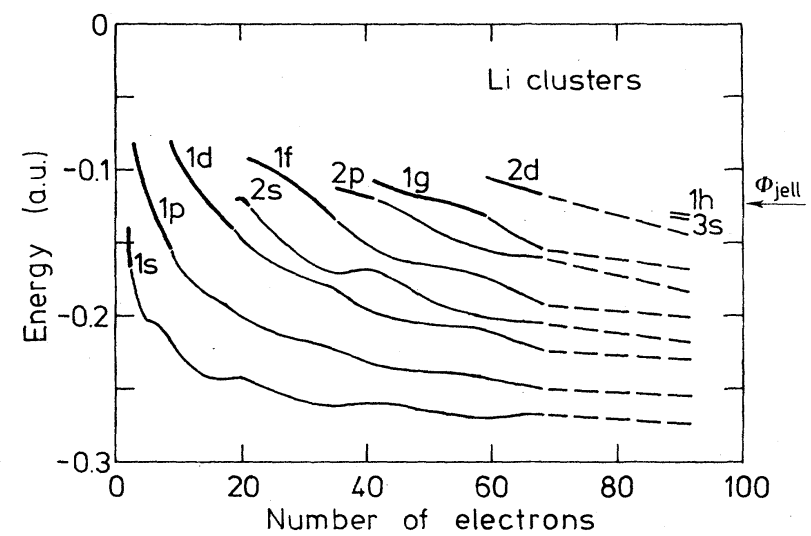

FIG. 1. One-electron Kohn-Sham eigenvalues for jellium spheres with $r_{s}=3.25 a_{0}(\mathrm{Li})$ as a function of the total number of electrons. The highest occupied level is denoted by a darker curve. $\Phi_{\text {jell }}$ shows the work function for the corresponding planar jellium surface. The dashed lines indicate a region where a self-consistent ground-state solution could not be determined (see text). act density functional calculation does give ${ }^{28}$ the correct ionization potential or work function. The local-density approximation becomes more and more appropriate as the eigenfunctions delocalize with the increase of the radius of the jellium sphere. The strong oscillatory behavior of the work function, according to Fig. 1, reflects, of course, the high degeneracy of the levels in the spherically symmetric system. In very small metal clusters the symmetry is lower, as high-symmetry clusters are not necessarily the stablest and most probably formed. ${ }^{29}$ However, there remains degeneracy in the electron states, which implies oscillatory size dependence of the work function. ${ }^{30}$ There is also experimental indication that for small sodium clusters the most stable ones are those which correspond to full-shell jellium clusters. ${ }^{10}$ We defer the further discussion of the relative stability of clusters of various sizes and shapes (the so-called magic numbers ${ }^{9,10,29}$ to a future publication. $^{31}$

Figure 2 shows the charge density and its components for the $\mathrm{Li}$ jellium sphere with 20 electrons. The electron density shows strong oscillations, resembling (but much larger than) the Friedel oscillations near a jellium surface. ${ }^{32}$ This pronounced deviation from the semi-infinite jellium, reflecting again the high degeneracy of the eigenstates in the spherical symmetry, persists to rather large sphere diameters. For example, in spheres with around 200 electrons, oscillations near the center are still remarkable. ${ }^{4,8}$ The size dependence of the density is especially strong at the sphere center, where only $s$ electrons contribute. Further from origin, the higher $l$ components superimpose, to follow somewhat more closely the Lang$\mathrm{Kohn}^{32}$ density on approach to the sphere surface. In the case presented in Fig. 2 the $2 s$ state has an important contribution near the surface of the sphere due to the orthogonalization against the $1 s$ state. This kind of rise in the electron density near the surface enhances the polarizability, as will be discussed below.

Figure 3 gives the energy level structure of the Li jellium sphere with 20 electrons. Bound states are represented by arrows, the lengths of which are proportional to the degeneracy. The continuum density of states $D(E)$ is given

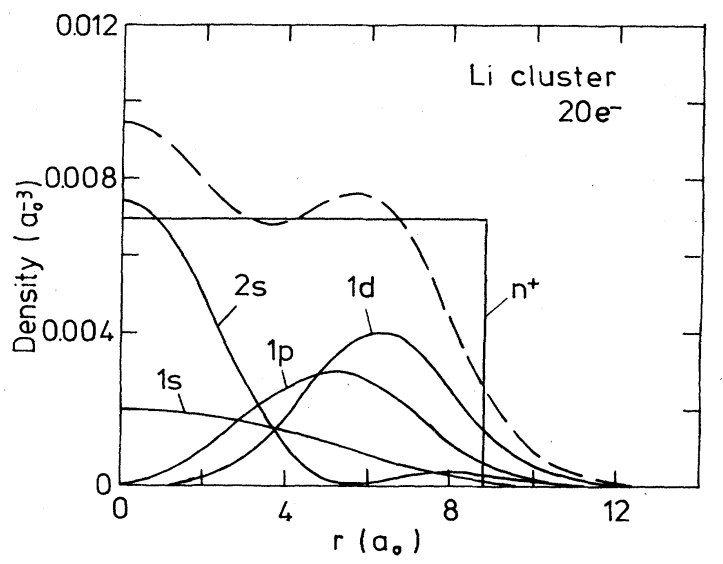

FIG. 2. The total electron density (dashed curve) and its decomposition in a jellium sphere with $r_{s}=3.25 a_{0}(\mathrm{Li})$ and containing 20 electrons. The homogeneous positive background charge $n^{+}$is also shown. 


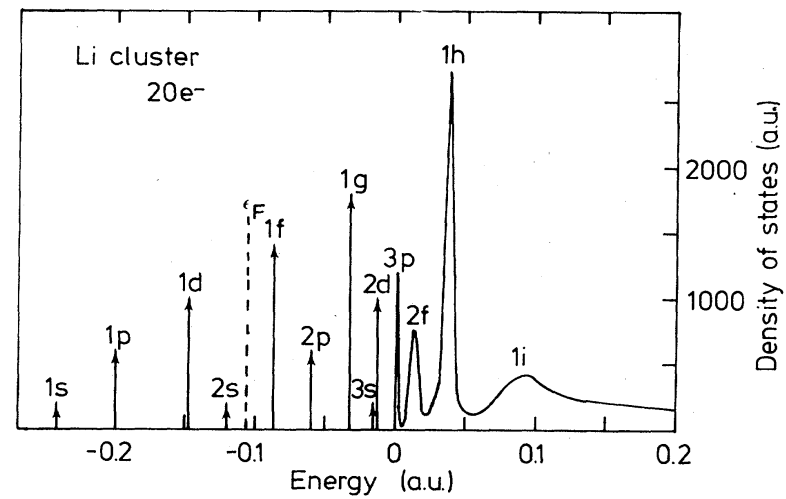

FIG. 3. One-electron ground state Kohn-Sham level structure for a 20-electron jellium sphere with $r_{s}=3.25 a_{0}$ (Li). Bound states at negative energies are denoted by arrows, the lengths of which are proportional to the degeneracies. Density of states, Eq. (25), is given for the positive energies, corresponding to the delocalized scattering states. The dashed line $\epsilon_{F}$ separates the occupied from unoccupied levels.

for positive energies corresponding to (delocalized) unoccupied states. $D(E)$ is calculated using the phase shifts $\delta_{l}(E)$ arising from the self-consistent potential $v_{\text {eff }}(r)$ as

$$
D(E)=\frac{2}{\pi} \sum_{i} \frac{\partial \delta_{l}(E)}{\partial E}(2 l+1) .
$$

The density of states shows remarkable peaks corresponding to resonant states behind the centrifugal potential barrier of the $l$ component in question. The widths of the peaks increase towards higher energies. This peak structure is reflected in the photoabsorption cross section above the bound to continuum edges (see below).

The total effective potential and its Coulomb part are shown in Fig. 4 for a Li jellium sphere with 20 electrons. The lower curves correspond to a sphere in vacuum $(\epsilon=1)$ and the upper ones to a jellium sphere embedded in

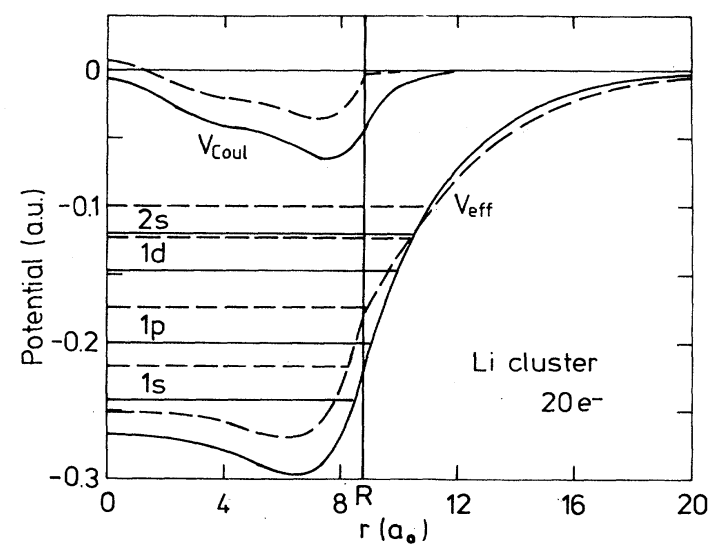

FIG. 4. Effective potential $V_{\text {eff }}$ and its Coulomb part for a 20-electron jellium sphere with $r_{s}=3.25 a_{0}$ (Li). Solid curves correspond to the sphere in vacuum $(\epsilon=1)$ and dashed curves show the potentials for a sphere surrounded by a dielectric medium with $\epsilon=20$. The occupied energy levels are also given. The jellium edge at $r=R$ is denoted by a vertical line. a dielectric medium with relative permeability $\epsilon=20$. It is seen that the exchange-correlation part of the effective potential dominates over the Coulomb part. The effect of the dielectric medium is taken into account through the solution [Eq. (6)] of the Poisson equation. Note that in the model the exchange-correlation potential is determined by the electron density arising from the occupied jellium-sphere states only, and any contribution due to the "bound electrons" in the dielectric medium are omitted. On the other hand, the exchange and correlation for $r>R$ are evaluated using the coupling constant 1 instead of $1 / \epsilon$, which would be implied by Eq. (6). The effective mass of electrons is also assumed to be unity throughout the space. In this approximation the effect of the dielectric medium is to shift rather rigidly the potential and the energy levels. The discontinuity of $d \phi / d r$ shifts the Coulomb potential at the surface of the sphere and decreases the surface dipole. The effect of the dielectric medium saturates rather quickly as $\epsilon$ increases.

\section{B. Static polarizabiilty}

We have calculated the static dipole polarizabilities for closed-shell $\mathrm{Li}$ and $\mathrm{Al}$ (jellium) spheres. In the case of $\mathrm{Li}$ we have also considered open-shell configurations using spin-independent density-functional theory and assuming spherical charge distributions also for the open shells. In order to test the validity of the approximations, we have first calculated the static polarizabilities of the second row atoms and compared them with other calculated ${ }^{33}$ and experimental $^{34}$ values in Table I. The order of magnitude and the trend along the row are well reproduced. The largest disagreement is seen in the case of alkaline atoms, for which the theoretical values are below the experimental ones. One reason for this is the local-density approximation which gives too loosely bound core states. This leads to the underestimation of the screening of the nuclear charge and as a consequence outer valence electrons are too tightly bound decreasing the polarizability. On the other hand, the calculations by Gollisch ${ }^{33}$ show that a spin-dependent treatment gives a relevant improvement over the spin-independent one.

Our results for the static polarizabilities of $\mathrm{Li}$ and $\mathrm{Al}$

TABLE I. Static polarizabilities of free atoms. The results of this work are obtained by using spin-independent densityfunctional theory and assuming spherical charge distributions for open shells. The values in Ref. 33 are obtained by a spindependent $X \alpha$ density matrix calculation. All values are in $a_{0}^{3}$.

\begin{tabular}{lccc}
\hline \hline Atom & This work & Ref. 33 & $\begin{array}{c}\text { Experiment } \\
\text { (Ref. 34) }\end{array}$ \\
\hline $\mathrm{Li}$ & 135 & 162 & 164 \\
$\mathrm{Be}$ & 42.9 & 40.9 & \\
$\mathrm{~B}$ & 23.9 & 23.8 & \\
$\mathrm{C}$ & 14.0 & 11.4 & \\
$\mathrm{~N}$ & 8.79 & 6.89 & 7.63 \\
$\mathrm{O}$ & 5.88 & 5.27 & 5.20 \\
$\mathrm{~F}$ & 4.12 & 3.78 & 2.67 \\
$\mathrm{Ne}$ & 3.00 & 2.77 & 159 \\
$\mathrm{Na}$ & 131 & 149 & \\
\hline \hline
\end{tabular}


jellium spheres are presented in Figs. 5 and 6. Calculated values are denoted by solid circles; the lines are drawn in order to guide the eye. The polarizabilities are given relative to the classical value $\alpha_{\text {class }}=R^{3}$ for a perfectly conducting metallic sphere (for a discussion of the definition of the radius, see below). The polarizability is enhanced over the classical value. The enhancement is largest for small spheres and the polarizability approaches the classical value rather slowly when the sphere size increases. The relative enhancement depends on the density of the positive background charge, increasing when the density increases. The enhancement is due to the spilling of the electron density out of the background sphere, as shown in Fig. 2. The discrete level structure of a quantummechanical calculation, on the other hand, tends to decrease the polarizability from the classical value, but this effect is more than compensated by the strong electronic relaxation. The oscillations of the polarizability as a function of sphere size are caused by the level structure. When a new level starts to fill, the polarizability is enhanced due to the large spatial extent of the new wave function. The polarizability then decreases as a function of the filling of the level because the wave functions become more and more localized. However, in some cases the orthogonality requirement against lower occupied states suppresses localization enough for the polarizability to actually increase when the level is being filled.

Ekardt $^{5}$ has calculated with similar techniques the polarizabilities for jellium spheres with $r_{s}=4$. His results are in agreement with ours. Before his work and the present one, the static polarizability of small jellium spheres was first calculated using the RPA by Rice et $a l .,{ }^{35}$ who employed the infinite barrier model for the surface of the sphere. This approach as well as others, ${ }^{17,19,36}$ where the electron density is not allowed to relax through a given sharp boundary, give a lower polarizability than the classical $R^{3}$. Note, however, that the question of enhancement or reduction of the polarizability over the classical value depends on the definition of the radius $R$. For example, in the jellium model the electron

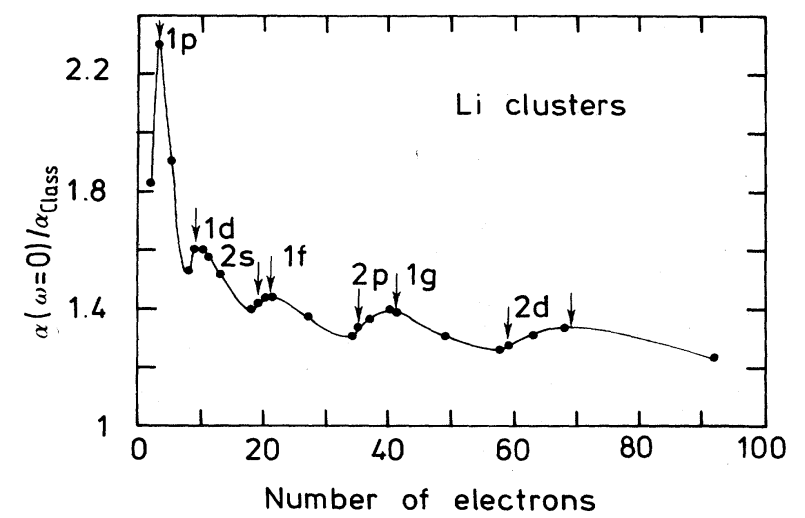

FIG. 5. Static $(\omega=0)$ polarizabilities of jellium spheres with $r_{s}=3.25 a_{0}$ (Li). The values are scaled by the classical result $\alpha_{\text {class }}=R^{3}$. The opening of new electron shells are denoted by arrows. The solid circles are the calculated values and the line is a guide to the eye.

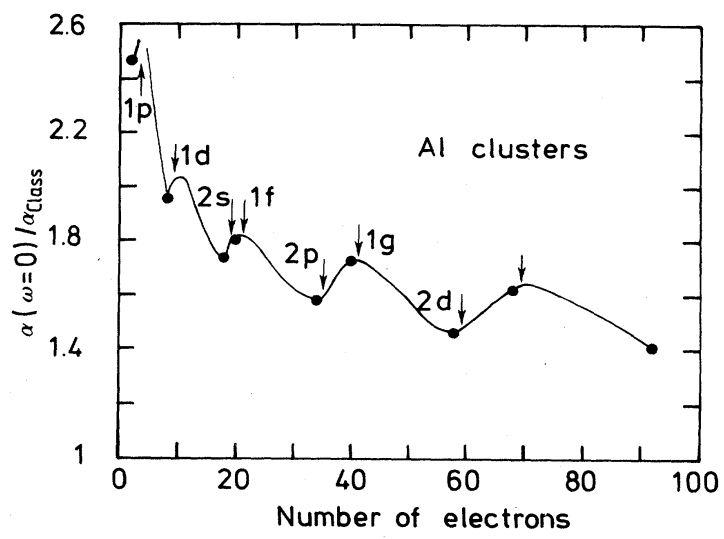

FIG. 6. Static $(\omega=0)$ polarizabilities of jellium spheres with $r_{s}=2.07$ (Al). The values are scaled by the classical result $\alpha_{\text {class }}=R^{3}$. The opening of new electron shells are denoted by arrows. The solid circles are the calculated values and the line is a guide to the eye.

density and the polarization charge (Fig. 2) reaches far outside the jellium edge, the sphere radius in this model. Consequently, for a given electron density (or polarization density) profile, the sphere radius of the jellium model is much smaller than that of the infinite barrier model. Thus results of these two models are then compared to different classical reference values. The different definition of the classical sphere radius also explains that in the earlier calculations the ratio $\alpha / \alpha_{\text {class }}$ decreases as the sphere radius decreases which is opposite to what we find in Figs. 5 and 6. Recently Snider and Sorbello ${ }^{6}$ have calculated the electron structure for a jellium sphere in an external field using a Thomas-Fermi-type densityfunctional method, which treats the kinetic energy functional as a gradient expansion of the electron density. A local-density approximation is used for exchange and correlation. Their model gives a polarizability which is also enhanced relative to the classical result $R^{3}$. They

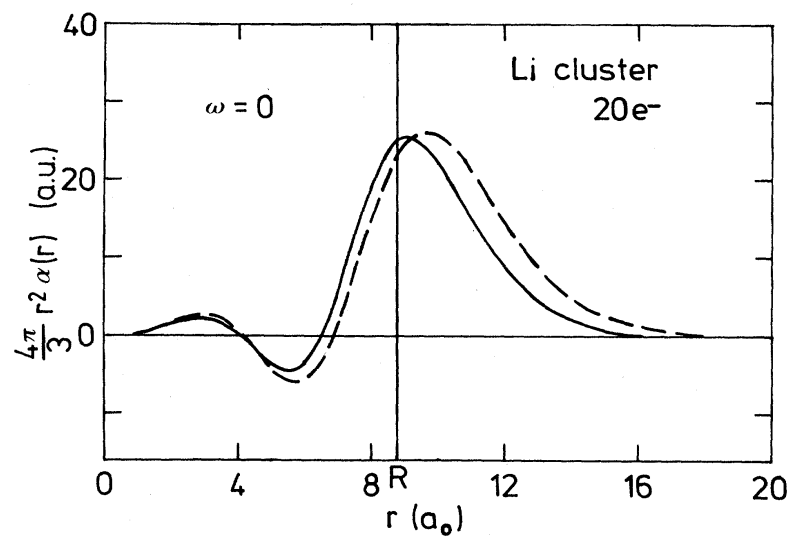

FIG. 7. Radial distribution of the induced dipole-moment density for a 20-electron jellium sphere with $r_{s}=3.25 a_{0}$ in a static field $(\omega=0)$. Solid and dashed lines correspond to a sphere in vacuum $(\epsilon=1)$ and a sphere in a dielectric medium with $\epsilon=20$, respectively. The position of the jellium edge at $r=R$ is also shown. 
write the polarizability in the form

$$
\alpha=(R+\delta)^{3} \text {, }
$$

where $\delta$ measures the effective increase of the sphere radius due to the relaxation of the electron density outside the positive background charge. According to Snider and Sorbello, $\delta$ is rather insensitive to the sphere radius. When compared to our results in Figs. 5 and 6, the data given by Snider and Sorbello show the same decreasing trend in $\alpha / \alpha_{\text {class }}$ when the sphere size increases, but their enhancement is considerably larger. The other difference is that the polarizabilities calculated by Snider and Sorbel$10^{6}$ do not show the level-structure oscillations due to approximation of kinetic energy by a density-gradient expansion.

Figure 7 shows the radial distribution of the static induced dipole-moment density $\frac{4}{3} \pi r^{2} \alpha(r)$ for the Li jellium sphere with 20 electrons. Most of the induced density is concentrated near the surface of the sphere, and the region outside the jellium edge is very important. This clearly explains how the previous models, in which the charge density is confined inside a hard wall sphere, give smaller polarizabilities, smaller than the classical result $\alpha_{\text {class }}=R^{3}$. This induced dipole-moment density has a counterpart in the calculations of Lang and $\mathrm{Kohn}^{37}$ for jellium surfaces. They calculated the induced charge density near the surface when a uniform perpendicular electric field is switched on. The resulting charge density near the surface and outside it is rather similar to the case of the jellium sphere in Fig. 7 and the profile for the sphere approaches the flat surface results when the sphere radius increases. However, although the approach is rather rapid for the surface region, the oscillations inside the sphere approach the Friedel oscillations of the flat surface much more slowly due to the discrete level structure in the jellium sphere.

Figure 8 shows the effect of the dielectric medium around the jellium sphere on the static polarizability. The polarizability increases and saturates rapidly as the dielectric constant of the medium increases. The saturation

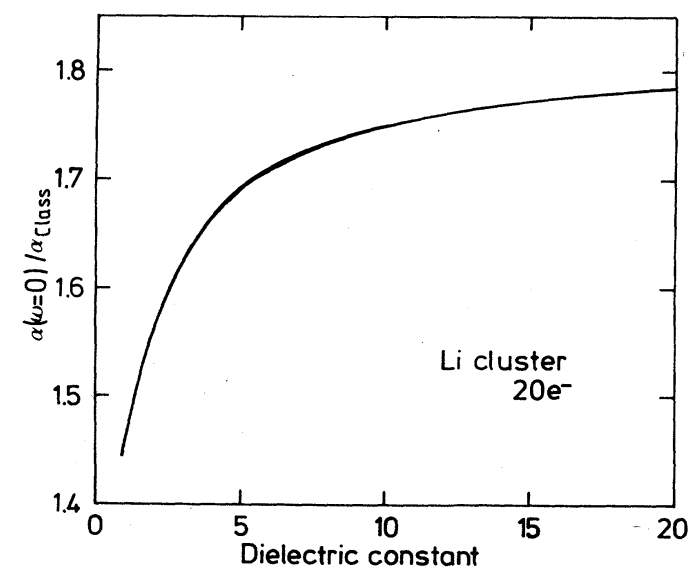

FIG. 8. Static polarizability of a jellium sphere with $r_{s}=3.25 a_{0}$ as a function of the dielectric constant $\epsilon$ of the surrounding medium. The values are scaled by the classical result $\alpha_{\text {class }}=R^{3}$. comes about as the Coulomb potential approaches zero outside the jellium sphere as shown in Fig. 4. We emphasize that the behavior of the polarizability in Fig. 8 is obtained using the model in which only the Coulomb part of the total effective potential is affected by the dielectric medium. For example, the physical exchange and correlation with the "bound" electrons in the dielectric medium would lower the potential outside the jellium sphere and increase the polarizability even further. It is interesting to note that the enhancement of the polarizabiilty by the embedding insulating medium is a quantum-mechanical effect in the sense that classical electrostatics predict no change in the induced dipole moment when a perfectly conducting sphere is placed in a dielectric.

\section{Dynamic polarizability}

We have calculated the response of $\mathbf{L i}$ jellium spheres with 2, 20, and 92 electrons to an alternating electromagnetic field as a function of the frequency $\omega$. For practical convenience, a small imaginary part, ${ }^{2} \Delta=0.0007$ a.u. $=10$ $\mathrm{meV}$ is introduced in the frequency $\omega$, as also suggested by Ekardt. ${ }^{5}$ This level broadening removes the singularities in the photoabsorption cross section calculated from Eq. (23), in a way which mimics finite-lifetime effects.

Figure 9 shows the photoabsorption cross section for a two-electron system. The dashed curve is calculated using the independent-particle-response function $\chi_{0}$, or equivalently, the two electrons respond independently to the external perturbation $v_{\text {ext }}$. The solid curve gives the cross section in the case where the electrons interact during the external perturbation, i.e., response function $\chi$ [Eq. (11)] is used [or, equivalently, the electrons respond independently to the change in the total effective potential (10)]. The peak in Fig. 9 is due to the $1 s-1 p$ particle-hole excitation. The peak has a finite height and width due to the imaginary part $\Delta$ in $\omega$. In the case of independent

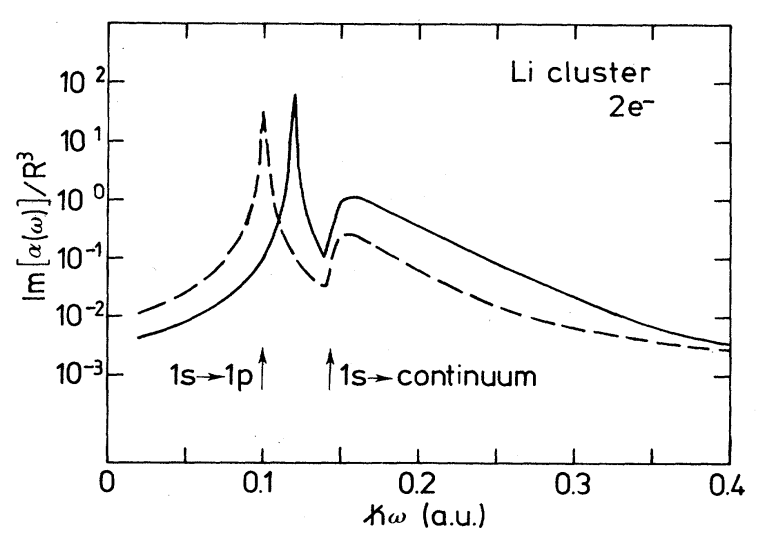

FIG. 9. Imaging part of the dynamic polarizability of a twoelectron jellium sphere as a function of the frequency $\omega$ of the driving field. The solid curve gives the interacting-electron result and the dashed curve corresponds to the independent response of electrons to the driving field. The imaginary part is directly proportional to the photoabsorption cross section [Eq. (23)]. The driving frequency $\omega$ has a constant imaginary part of $\hbar \Delta=10 \mathrm{meV}$. The position of the $1 s 2 p$ energy differences is given as well as the distance from the $1 s$ level to the continuum. 
response the absorption peak is exactly at the energy corresponding to the difference of the Kohn-Sham oneparticle energy levels. The interacting electron peak is shifted to higher energies. This shift originates as follows. When the energy argument $\epsilon_{i}+\omega$ of the Green function in Eq. (18) is equal to the Kohn-Sham eigenvalue for a level with angular quantum number $l_{i} \pm 1$, the solutions $j$ and $h$ are linearly dependent and the Wronskian determinant in the denominator of the Green's function vanishes. This leads to a sharp absorption peak. When $\omega$ has an imaginary part, the Wronskian near the resonance becomes small but remains nonzero. The response function for the interacting system is calculated by solving a matrix equation [corresponding to Eq. (11)]. The increase of the Green's function increases the elements of the vector on the right-hand side of the equation, but at the same time the elements of matrix to be inverted increase. Thus there is a competition situation, which leads to maximum absorption at an energy differing from the energy difference of the eigenstates. The $1 s$-continuum edge is another prominent feature of Fig. 9. The edge is smoothened due to the imaginary part of $\omega$, but is not moved by electronelectron interactions.

Besides the $1 s-1 p$ peak there exist no other boundbound particle-hole excitations for the two-electron sphere. The Rydberg levels before the continuum edge are missing in the local-density approximation due to the exponential decay of the effective potential. However, as discussed by Zangwill and Soven, ${ }^{26}$ this missing absorption channel is partly compensated by the lowering of the bound-continuum edges in the local-density approximation and the Kramers-Kronig relation, Eq. (24), for the integral of the cross-section curve becomes thereby fulfilled.

Figure 10 shows the calculated photoabsorption cross sections for the Li jellium sphere with 20 electrons. Now there are several particle-hole excitation modes corresponding to the level structure of Fig. 3. Especially, it is interesting to note that transitions from $2 s$ to $1 d$ levels to $p$ - and $f$-like resonances above zero energy produce rather broad humps above the maximum peak of $\operatorname{Im}[\alpha(\omega)]$. The bound-bound cusps and the bound-continuum edges superimpose in the interacting electron case to form a broad structure around $\omega=1.4$ a.u. In the independent electron

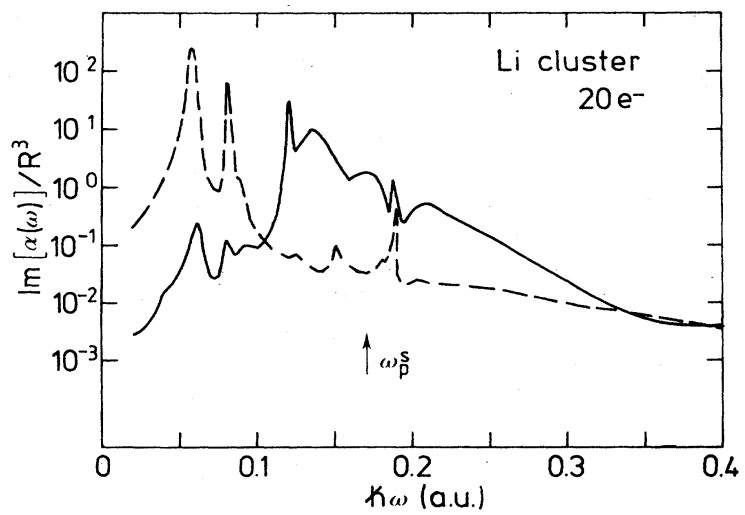

FIG. 10. Same as Fig. 9 but for a 20-electron jellium sphere with $r_{s}=3.25 a_{0}$. The position of the classical surface plasmon $\omega_{p}^{s}$ is also given. case the large absorption occurs at lower frequencies. In the intermediate frequency region the absorption cross section is, apart from some cusps, a rather flat function of frequency. These tendencies are even more pronounced in the case of a jellium sphere with 92 electrons, shown in Fig. 11. For the interacting electron system the broad region of large absorption around $\omega=1.4$ a.u. can be attributed to a collective mode, which eventually will become the surface plasmon. Above all bound-continuum edges around $\omega=0.33$ a.u., the cross section has a smooth hump. This increase in the absorption cross section has to be due to collective effects, because the independentelectron model does not show this kind of structure. We assign this increase in the absorption as an emerging bulk plasmon mode, which is slightly blue-shifted from the classical value.

Figure 11 also shows the photoabsorption cross section obtained from the classical (Drude) theory. According to classical electrostatics, the polarizability of a sphere of dielectric constant $\epsilon_{m}$ in an external electric field is

$$
\alpha=R^{3}\left(\frac{\epsilon_{m}-1}{\epsilon_{m}+2}\right) .
$$

The use of the Drude model,

$$
\epsilon_{m}(\omega)=1-\frac{\omega_{p}^{2}}{\omega(\omega+i / \tau)},
$$

where $\omega_{p}$ is the plasma frequency and $\tau$ is the scattering (relaxation) time, leads to a photoabsorption cross section proportional to

$$
\operatorname{Im}[\alpha(\omega)]=R^{3} \omega_{p}^{2} \frac{3 \omega / \tau}{\left(\omega_{p}^{2}-3 \omega^{2}\right)^{2}+(3 \omega / \tau)^{2}} .
$$

This has a maximum at $\omega_{s}=\omega_{p} / \sqrt{3}$, which is the surface plasmon frequency. The classical curve in Fig. 11 is drawn using a value for the relaxation time $\tau$ consistent with the $10-\mathrm{meV}$ imaginary part in $\omega$. In principle, $\tau$ should describe both electron scattering (lifetime effects) in bulk and the additional surface scattering due to the finite size of the sphere. ${ }^{18}$ The self-consistent solution for

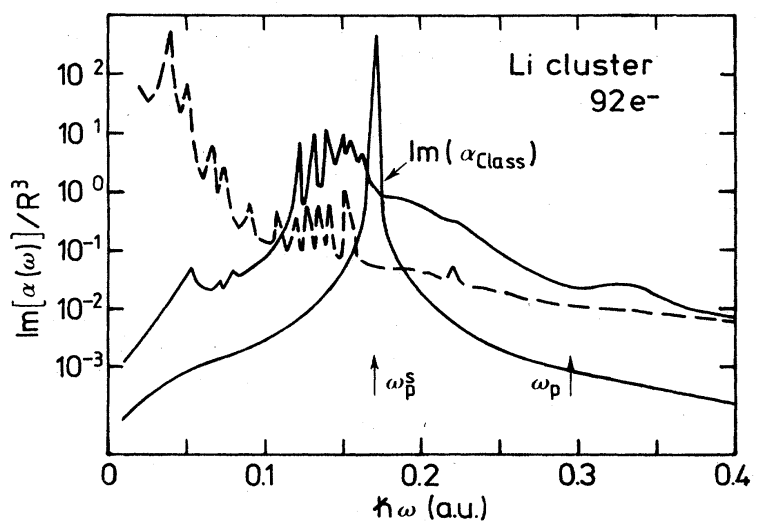

FIG. 11. Same as Fig. 9 but for a 92-electron jellium sphere with $r_{s}=3.25 a_{0}$. The positions of the classical surface plasmon $\omega_{p}^{s}$ and bulk plasmon $\omega_{p}$ are denoted by arrows. The classical result, Eq. (28), is also given. 


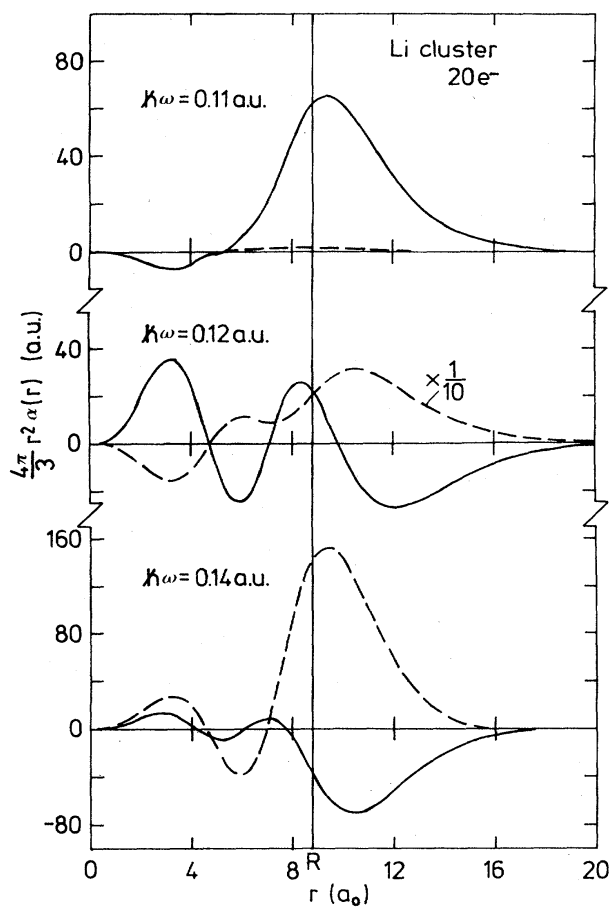

FIG. 12. Radial distribution of the induced dipole-moment density for a 20-electron jellium sphere with $r_{s}=3.25 a_{0}$ at three different driving field frequencies near the maximum photoabsorption. Real and imaginary parts are given by solid and dashed curves, respectively. The frequency has an imaginary part of $\hbar \Delta=10 \mathrm{meV}$.

the photoabsorption cross section is strongly deviant from the classical behavior. The contribution due to boundbound transitions is important. Moreover, the surfaceplasmon peak is shifted towards to lower energies from the classical value 0.171 a.u. and is strongly broadened. This broadening as well as the red-shift is in accord with the theory of Wood and Ashcroft. ${ }^{18}$ The red-shift of the surface-plasmon frequency is in qualitative agreement with the experimental results of Smithard et al. for sodi$\mathrm{um}^{38}$ and silver ${ }^{39}$ spheres but opposite to the results of Genzel and Kreibig ${ }^{40}$ for silver.

Ekardt ${ }^{5}$ has calculated the photoabsorption cross section for a jellium sphere with $r_{s}=4$ a.u. and containing 192 electrons. His results, in particular the shifts of the collective modes are in qualitative agreement with our results in Fig. 11.

The spatial behavior of the real and imaginary parts of the induced-dipole moment density around the maximum absorption is given in Fig. 12 in the case of a jellium sphere with 20 electrons. Just before the maximum of $\alpha(\omega)$ at $\omega=0.11$ a.u. the real part is larger than the zero- frequency value in Fig. 7 by a factor of 3, but the imaginary part nearly vanishes. A small increase in the frequency to $\omega=0.12$ a.u. results in a large increase in the imaginary part and the real part is seen to oscillate in a complicated manner. After the maximum absorption at $\omega=0.14$ a.u., the real part becomes negative at large distances, i.e., the charge density is oscillating out of phase relative to the driving field. A similar behavior is seen also by Zangwill and Soven ${ }^{26}$ in the case of photoabsorption by inert-gas atoms.

\section{CONCLUSIONS}

We have presented results of a comprehensive calculation of the static and dynamic properties of microscopically small metallic spheres. While the basic model of the spheres, the jellium mode, is a crude one, it nevertheless contains many of the essential features of a more realistic molecular approach. On the other hand, the model problem is solved basically exactly using the time-dependent density-functional method. The only additional approximations are involved in the construction of exchangecorrelation energy functional, a topic of substantial current interest.

The results of the fully self-consistent calculations show interesting deviations from the approximate solutions to the same problem. Size effects show up in the groundstate electronic properties in a manner now well understood. The static polarizability is enhanced over the classical value, the more so the smaller the cluster. If the metallic particles are embedded in a dielectric medium, the polarizability will again be enhanced, contrary to the classical expectations.

The dynamic response is particularly interesting. The results clearly show the importance of single-particle excitations, including both bound-bound and boundcontinuum transitions for the particle-hole pairs. Moreover, the emergence of collective modes can be monitored and their shifts from classical values are obtained. These exact results should prove very useful in constructing approximate expressions (such as the hydrodynamic one) for modeling the response. These will be necessary in particular for large clusters $(N>500)$ where the calculations of the present type quickly become unmanageable.

Finally, the results give an unambiguous starting point for model calculations of a number of processes, which show seemingly anomalous behavior (typically large enhancements) in small metal particles: the photoyield, ${ }^{14}$ van der Waals forces, ${ }^{15}$ Raman scattering, ${ }^{41}$ etc. Howev-. er, it seems that while a fully self-consistent and nonlocal treatment can give substantial deviations from classical behavior, it alone cannot explain the enormously large enhancements sometimes observed.
${ }^{1}$ For a review, see J. A. A. J. Perenboom, P. Wyder, and F. Meier, Phys. Rep. 78, 173 (1981).

${ }^{2}$ S. Doniach, in Many-Body Phenomena at Surfaces, edited by D. Langreth and H. Suhl (Academic, New York, 1984), p. 241.
${ }^{3}$ R. P. Devaty and A. J. Sievers, Phys. Rev. Lett. 52, 1344 (1984).

${ }^{4}$ W. Ekardt, Phys. Rev. B 29, 1558 (1984).

${ }^{5}$ W. Ekardt, Phys. Rev. Lett. 52, 1925 (1984).

${ }^{6}$ D. R. Snider and R. S. Sorbello, Phys. Rev. B 28, 5702 (1983); 
Solid State Commun. 47, 845 (1983); Surf. Sci. 140, 204 (1984); see also M. Cini, J. Catal. 37, 187 (1975).

${ }^{7}$ R. S. Sorbello, Solid State Commun. 48, 989 (1983).

${ }^{8}$ D. E. Beck, Solid State Commun. 49, 381 (1984).

${ }^{9}$ C. Baladron, M. P. Iniquez, and J. A. Alonso, Solid State Commun. 50, 549 (1984); Surf. Sci. 127, 367 (1983).

${ }^{10}$ W. D. Knight, K. Clemenger, W. A. de Heer, W. A. Sanders, M. Y. Chou, and M. L. Cohen, Phys. Rev. Lett. 52, 2141 (1984).

${ }^{11}$ D. R. Penn and R. W. Rendell, Phys. Rev. Lett. 47, 1067 (1981).

12P. Apell and D. R. Penn, Phys. Rev. Lett. 50, 1316 (1983).

${ }^{13}$ P. Apell, Phys. Scr. 29, 146 (1984).

${ }^{14}$ A. Schmidt-Ott, P. Schurtenberger, and H. C. Siegmann, Phys. Rev. Lett. 45, 1284 (1980).

${ }^{15}$ H. Burtscher and A. Schmidt-Ott, Phys. Rev. Lett. 48, 1734 (1982).

16D. B. Tran Thoai and W. Ekardt, Solid State Commun. 41, 687 (1982).

${ }^{17}$ W. Ekardt, D. B. Tran Thoai, F. Frank, and W. Schulze, Solid State Commun. 46, 571 (1983).

${ }^{18}$ D. M. Wood and N. W. Ashcroft, Phys. Rev. B 25, 6255 (1982).

${ }^{19}$ B. B. Dasgupta and R. Fuchs, Phys. Rev. B 24, 554 (1981).

${ }^{20}$ W. Kohn and L. J. Sham, Phys. Rev. 140, 1133A (1965).

${ }^{21}$ For a recent review, see Theory of the Inhomogeneous Electron Gas, edited by S. Lundqvist and N. H. March (Plenum, New York, 1983).

${ }^{22}$ M. J. Stott and E. Zaremba, Phys. Rev. A 21, 12 (1980).

23P. J. Feibelman, Phys. Rev. B 12, 1319 (1975); 12, 4282 (1975); Prog. Surf. Sci. 12, 287 (1982).

${ }^{24}$ O. Gunnarsson and B. I. Lundqvist, Phys. Rev. B 13, 4274 (1976).

${ }^{25}$ P. Ziesche and D. Lehmann, J. Phys. C 15, 4807 (1982); 16,
879 (1983), and unpublished.

${ }^{26}$ A. Zangwill and P. Soven, Phys. Rev. A 21, 1561 (1980).

27J. F. Janak, V. L. Moruzzi, and A. R. Williams, Phys. Rev. B 12, 1257 (1975).

28J. P. Perdew and M. Levy, Phys. Rev. Lett. 20, 1884 (1983). See also Ref. 21.

${ }^{29}$ J. L. Martins, J. Buttet, and R. Car, Phys. Rev. Lett. 53, 655 (1984).

${ }^{30}$ A. Hermann, E. Schumacher, and L. Wöste, J. Chem. Phys. 68, 2327 (1978).

${ }^{31}$ P. Jena, B. Rao, M. Manninen, M. J. Puska, and R. M. Nieminen (unpublished).

${ }^{32}$ N. D. Lang and W. Kohn, Phys. Rev. B 1, 4555 (1970).

${ }^{33}$ H. Gollisch, J. Phys. B 17, 1463 (1984).

${ }^{34}$ The experimental polarizabilities are from the following sources: Li and Na, R. W. Molet, H. L. Schwartz, T. M. Miller, and B. Bederson, Phys. Rev. A 10, 1131 (1974); Ne, R. R. Teachout and R. T. Pack, At. Data 3, 195 (1971); N and O, R. A. Alpher and D. R. White, Phys. Fluids 2, 1953 (1959).

${ }^{35}$ M. J. Rice, W. R. Schneider, and S. Strässler, Phys. Rev. B 8, 474 (1973); S. Strässler, M. J. Rice, and P. Wyder, Phys. Rev. B 6, 2575 (1972).

${ }^{36}$ A. A. Lushnikov and A. J. Simonov, Z. Phys. 270, 17 (1974).

${ }^{37}$ N. D. Lang and W. Kohn, Phys. Rev. B 7, 3541 (1973).

${ }^{38}$ M. A. Smithard and M. Q. Tran, Helv. Phys. Acta 46, 869 (1974).

${ }^{39}$ M. A. Smithard, Solid State Commun. 13, 153 (1973); J. D. Grariere, R. Rechsteiner, and M. A. Smithard, ibid. 16, 113 (1975).

${ }^{40}$ L. Genzel and U. Kreibig, Z. Phys. B 37, 93 (1980).

41J. A. Creighton, C. G. Blatchford, and M. G. Albrecht, J. Chem. Soc. Faraday Trans. 2 75, 790 (1979). 